\title{
A Study on a Magnesium-Based Layered Composite Used as a Flame Retardant for Phenolic Epoxy Resins
}

\author{
Hongxiang Liu ${ }^{1,2,}$, Neng Xiong ${ }^{1,2}$, Songli Wang ${ }^{1,2}$, Wei Zhang ${ }^{1,2}$ and BoYong ${ }^{1,2}$ \\ ${ }^{1}$ National Engineering Laboratory for Vacuum Metallurgy, Key Laboratory of Nonferrous Metals Vacuum Metallurgy of Yunnan \\ Province, Kunming, 650093, China \\ ${ }^{2}$ Faculty of Metallurgical and Energy Engineering, Kunming University of Science and Technology, Kunming, 650093, China \\ *Corresponding Author: Hongxiang Liu. Email: 20203102002@stu.kust.edu.cn
}

Received: 20 June 2021 Accepted: 20 August 2021

\begin{abstract}
The effects of a magnesium-based layered composite on the flammability of a phenolic epoxy resin (EP) are studied. In order to produce the required composite material, first, magnesium hydroxide, aluminum salt and deionized water are mixed into a reactor according to a certain proportion to induce a hydrothermal reaction; then, the feed liquid is filtered out using a solid-liquid separation procedure; finally, the material is dried and crushed. In order to evaluate its effects on the flammability of the EP, first, $\mathrm{m}$-phenylenediamine is added to EP and vacuum defoamation is performed; then, EP is poured into a polytetrafluoroethylene mold, cooled to room temperature and demoulded; finally, the magnesium-based layered composite is added to EP, and its flame retardance is characterized by thermogravimetric analysis, limiting oxygen index and cone calorimetry. The X-ray diffraction patterns show that the baseline of magnesium-based layered composite is stable and the front shape is sharp and symmetrical when the molar ratio of magnesium to aluminium is $3.2: 1$; with the addition of magnesium-based layered composite, the initial pyrolysis temperature of EP of $10 \%, 15 \%$ and $30 \%$ magnesium-based layered composite decreases to $318.2^{\circ} \mathrm{C}, 317.9^{\circ} \mathrm{C}$ and $357.1^{\circ} \mathrm{C}$, respectively. After the reaction, the amount of residual carbon increases to $0.1 \%, 3.45 \%$ and $8.3 \%$, and the limiting oxygen index increases by $28.3 \%, 29.1 \%$ and $29.6 \%$, respectively. The maximum heat release rate of cone calorimeter decreases gradually. The optimum molar ratio of $\mathrm{Mg}: \mathrm{Al}$ for green synthesis is 3.2:1, and the NO3- intercalated magnesium-based layered composite has the best flame retardance properties.
\end{abstract}

\section{KEYWORDS}

Phenolic epoxy resin; layered composite; green synthesis; flame retardance; anion intercalation

\section{Introduction}

Layered double hydroxides (LDHs) are layered materials composed of positively charged main lamina, negatively charged interlayer anions and interlayer water molecules, also known as hydrotalcite or hydrotalcite-like compounds [1]. LDHs can use its layered compound host to introduce some functional guest substances into the interlayer gap under the action of strong polar molecules and expand the laminate distance, so as to form a layered host compound. LDHs have the characteristics of adjustable chemical composition of main laminates, adjustable number and types of guest anions between layers, 
and adjustable particle size distribution of intercalation assemblies. LDHs can provide good flame retardance and smoke suppression performance due to their unique chemical composition and layered structure, which is expected to become a potential environmental-friendly flame retardant.

The synthetic process of LDHs mainly includes coprecipitation, hydrothermal synthesis, ion exchange, roasting recovery and so on [2]. Among them, the most commonly used method is coprecipitation. Coprecipitation method is to mix alkali solution with salt solution to make the mixed solution undergo coprecipitation reaction, so as to obtain colloid, and then react the colloid at a certain temperature and time to finally obtain LDHs. In the coprecipitation method, massive water resources need to be adopted to adjust the $\mathrm{pH}$ of alkali solution and salt solution. impurities are easy to form during the experiment due to the continuous change of $\mathrm{pH}$ value. Specifically, the synthesis methods of magnesium-based LDHs mainly use soluble magnesium salts $\left(\mathrm{MgCl}_{2}, \mathrm{Mg}\left(\mathrm{NO}_{3}\right)_{2}\right.$ and $\left.\mathrm{MgSO}_{4}\right)$ as raw materials [3], and some insoluble magnesium salts (such as $\mathrm{MgO}$ and $\mathrm{Mg}(\mathrm{OH})_{2}$ ) are also reported as raw materials. However, massive water resources are wasted in the traditional process, leading to a large amount of waste residues polluting environment [4]. Hence, a new green synthesis process of MgAl-LDHs is evaluated by using $\mathrm{Mg}(\mathrm{OH})_{2}$ as raw material.

Phenolic epoxy resin (EP) is a kind of organic compound with two or more epoxy groups in molecular structure [5]. It has a wide application in daily life, while its flammability brings multiple potential safety hazards [6]. Current flame retardancy studies involve intercalation cations of LDHs, interlayer inorganic anions of LDHs, interlayer organic anions and surface modification [7]. Based on the two aspects of green synthesis MgAl-LDHs addition and anion intercalation, three characterization methods including thermogravimetric analysis, limiting oxygen index (LOI) and cone calorimetry are adopted to evaluate the effect of MgAl-LDHs on the flame retardance of EP [8]. Thermogravimetric analysis is mainly employed to study the functional relationship between the weight and energy of polymer materials and temperature, which can accurately measure the mass change and change rate of materials. It is widely adopted to evaluate the thermal stability of polymer materials and the effect of additives on thermal stability; the LOI method is good for quantitative analysis and is mainly employed to evaluate the combustion performance of plastics, rubber and other materials; cone calorimetry is one of the most crucial characterization methods to evaluate the combustion performance of polymer materials.

To deal with the characteristics of easy combustion of EP, green synthetic magnesium-based layered composite is introduced to EP for flame retardant. The green synthesis of magnesium-based layered composite is carried out by introducing the characteristics of magnesium-based layered composite and the evaluation method of their flame retardant properties. On this basis, thermogravimetric analysis, LOI and cone calorimetry are adopted to study the flame retardant properties of magnesium-based layered composite. The innovation is to combine the flame retardant research of green synthetic magnesiumbased layered composite with EP, obtain the best process ratio of green synthetic magnesium-based layered composite, and analyze the flame retardant effect of magnesium-based layered composite on EP. The green synthesis of magnesium-based layered composite is adopted. The preparation conditions are simple and friendly to the environment. The magnesium-based layered composite is added to EP, and its flame retardance is evaluated, which has a certain reference value for the flame retardance application of this kind of product.

\section{Introduction of Research Background}

\subsection{Synthesis of Layered Composites}

LDHs has been widely adopted in multiple fields because of its easily adjustable structure and composition. However, its reserves in nature are small and difficult to meet industrial requirements, leading to the technological development of synthetic LDHs. The common synthesis methods of LDHs include baking recovery method, hydrothermal synthesis method, coprecipitation method and deuterium 
exchange method. Baking recovery method is suitable for the preparation of anionic intercalated hydrotalcite, mainly using the unique structure and properties of LDHs. The baking recovery method bakes LDHs at $500^{\circ} \mathrm{C}$ to obtain composite metal oxides because the material has good memory effect, which are placed in anionic solution. At this time, the target anion will enter the layered structure of LDHs; hydrothermal synthesis takes hydroxide and oxide as raw materials, and the laminate generally contains $\mathrm{M}^{2+}$ and $\mathrm{M}^{3+}$. The experimental conditions are high pressure and high temperature. The experimental raw materials include magnesium oxide, magnesium hydroxide, aluminum hydroxide, and alumina. Finally, LDHs with good crystal form are obtained; coprecipitation method is to mix alkali solution and salt solution to obtain colloid after coprecipitation effect, and then react the colloid at a certain temperature and time to finally obtain LDHs; deuterium exchange method also uses the interlayer anion tunability of LDHs to obtain LDHs.

The above traditional methods usually use soluble magnesium salt as raw material in the process of product preparation, which often wastes massive water resources and generates certain by-products that is harmful to the environment. Therefore, the current LDHs preparation is mostly to find a more green and feasible preparation method from the perspective of environment. Insoluble magnesium salt is one of the hot research topics. In some studies, Mg-LDHs with good crystal form are obtained from alumina and magnesium oxide based on hydrothermal synthesis. There are also studies on the preparation of LDHs under high pressure based on magnesium hydroxide, aluminum hydroxide and ammonium carbonate, and a green preparation method without by-products is obtained.

\subsection{Introduction to $\mathrm{EP}$}

EP is the general name of polymers containing two or more epoxy groups. It is the polycondensation product of epichlorohydrin with bisphenol A or polyhydric alcohols. The classification of EP has not been unified at present. The general classification is based on the characteristics such as heat resistance grade and strength. Fig. 1 displays the chemical structure of bisphenol A EP.

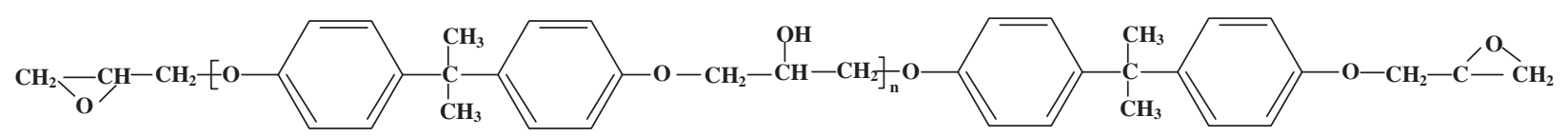

Figure 1: Chemical structure of EP

Fig. 1 shows that bisphenol A EP has four epoxy groups. The epoxy group is easy to react with active ammonia and hydrogen, while the curing agent contains active hydrogen and ammonia. Hence, EP will have cross-linking reaction with the curing agent, and finally form a three-dimensional network structure. The LOI of EP is about $20 \%$, which is flammable in case of fire, and the epoxy tree will emit massive heat and smoke during combustion, so it is easy to cause property loss and casualties.

\subsection{Research Progress on Flame Retardancy of Layered Composites}

LDHs have been studied in the field of flame retardant because of its unique layered structure. As an organic flame retardant, LDHs have better flame retardancy than inorganic flame retardants such as magnesium hydroxide and aluminum hydroxide. The thermal decomposition of LDHs can be divided into the removal of interlayer water molecules, the decomposition of interlayer hydroxyl and interlayer anions, and the emergence of composite metal oxides. LDHs mainly rely on the absorption of heat in thermal decomposition to reduce the combustion temperature. The removal of interlayer water molecules and anions of LDHs can take away massive heat and cool down. The addition process of LDHs can accelerate the carbonization of polymer materials and block the diffusion of combustible gas. 
In the current research, the interlayer cations of LDHs can promote the flame retardant properties of LDHs. The flame retardant effect of LDHs interlayer inorganic anions is as follows: nitrate ion $>$ sulfate ion $>$ carbonate ion $>$ chloride ion. The addition of interlayer organic anions can significantly improve the carbon formation of the composites. The addition of inorganic fillers also plays a positive role in the flame retardancy of LDHs. With the development of interdisciplinary, the current research on the flame retardant properties of LDHs begins to explore the synergistic flame retardant effect of LDHs and other flame retardants.

Based on the above contents, the green synthesis of magnesium-based layered composites take insoluble magnesium salts as raw materials. On this basis, it is combined with inorganic anion intercalation, and the flame retardant properties of magnesium-based layered composites in EP are studied.

\section{Green Synthesis of Magnesium-Based Layered Composite and Evaluation of Their Flame Retardance}

\subsection{Green Synthesis Method of Magnesium-Based Layered Composite}

(1) Experimental materials and instruments

The experimental materials used in the research process included magnesium hydroxide $\left(\mathrm{Mg}(\mathrm{OH})_{2}\right)$, aluminum chloride hexahydrate $\left(\mathrm{AlCl}_{3} \cdot 6 \mathrm{H}_{2} \mathrm{O}\right)$, aluminum nitrate nonahydrate $\left(\mathrm{Al}\left(\mathrm{NO}_{3}\right)_{3} \cdot 9 \mathrm{H}_{2} \mathrm{O}\right)$, aluminum sulfate octadecahydrate $\left(\mathrm{Al}_{2}\left(\mathrm{SO}_{4}\right)_{3} \cdot 18 \mathrm{H}_{2} \mathrm{O}\right)$ and deionized water $\left(\mathrm{H}_{2} \mathrm{O}\right)$. The deionized water is made in the laboratory, and its conductivity is $\leq 10^{-6} \mathrm{~S} \cdot \mathrm{cm}^{-1}$. The rest of the raw materials are from Sinopharm Chemical Reagent Co., Ltd. (Shanghai, China) with analytical purity.

The instruments used in the research process included BSA224S-CW electronic analytical balance (produced by Sartorius Instrument Co., Ltd. (Beijing, China)), DF-101S constant temperature magnetic stirrer (produced by Gongyi Yuhua Instrument Co., Ltd. (Gongyi, China)), GZX-9070MBE electric blast drying oven (produced by Shanghai Boxun Industrial Co., Ltd. (Shanghai, China)), LD5-2B centrifuge (produced by Beijing Jingli centrifuge Co., Ltd. (Beijing, China)), LD-DI-Micro ultra-pure water instrument (produced by Shanghai Liding water treatment equipment Co., Ltd.), and X'pert Pro X-ray diffractometer (PANalytical Instrument Co., Ltd. (Malvern, UK)).

(2) Experimental methods

Fig. 2 is the synthesis process flow of MgAl-LDHs.

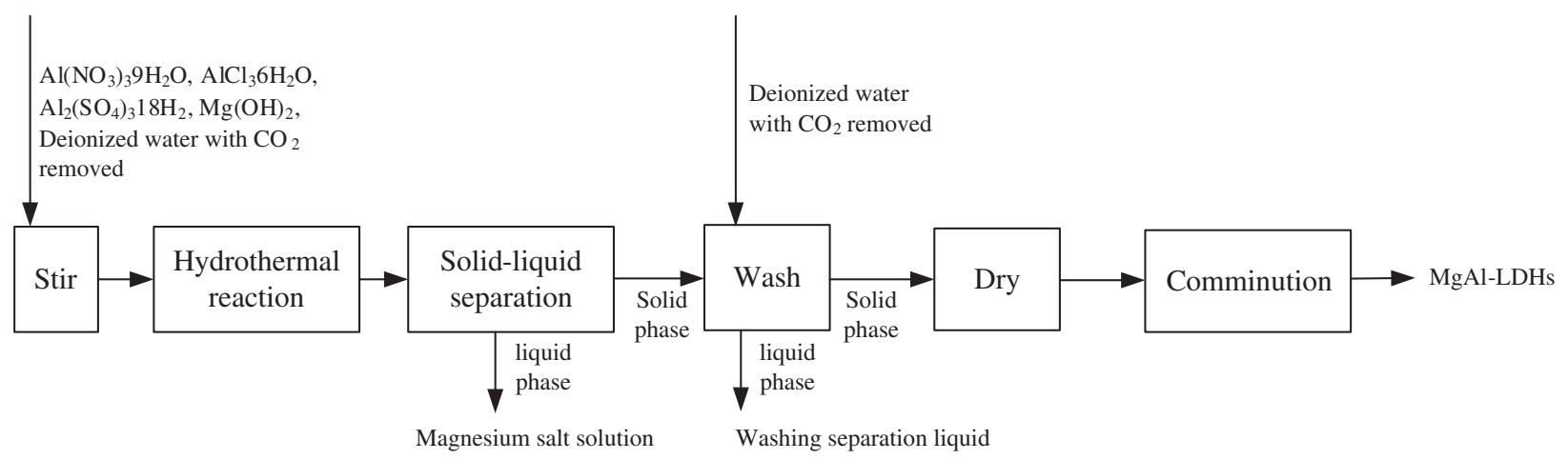

Figure 2: Synthesis process flow of MgAl-LDHs

Fig. 2 displays that the synthesis process of $\mathrm{MgAl}$ LDHs is mainly as follows. $0.7213 \mathrm{Mg}(\mathrm{OH})_{2}$ was weighed. The corresponding $\mathrm{Al}\left(\mathrm{NO}_{3}\right)_{3} \cdot 9 \mathrm{H}_{2} \mathrm{O}, \mathrm{AlCl}_{3} \cdot 6 \mathrm{H}_{2} \mathrm{O}$ and $\mathrm{Al}_{2}\left(\mathrm{SO}_{4}\right)_{3} \cdot 18 \mathrm{H}_{2} \mathrm{O}$ were weighed according to the ratio of $\mathrm{Mg}: \mathrm{Al}=3: 1 \sim 4: 1$. Aluminum salt and $\mathrm{Mg}(\mathrm{OH})_{2}$ were added into $120 \mathrm{~mL}$ of deionized water with $\mathrm{CO}_{2}$ removed and stirred vigorously for $120 \mathrm{~min}$. The mixture liquid was transferred to the 
reactor and reacted at different temperatures and time; after the reaction, the slurry was centrifuged at the speed of $8000 \mathrm{r} \cdot \mathrm{min}^{-1}$, separated, washed, and then dried in a $90^{\circ} \mathrm{C}$ vacuum drying oven for $12 \mathrm{~h}$ to obtain MgAl-LDHs products.

(3) Experimental characterization means

X-ray diffraction (XDR) was mainly adopted to characterize the crystal structure of the samples [9]. Sample pretreatment: the dried sample was ground into the powder with a mortar, and then the tablet was pressed into the mold. Test conditions: $\mathrm{Cu}$ target, $\mathrm{Ka}$ ray, $\lambda=14573$, tube voltage $40 \mathrm{kV}$, tube current $30 \mathrm{~mA}$, test range $2 \theta=20^{\circ} \sim 80^{\circ}$, and test speed $5^{\circ} \cdot \mathrm{min}^{-1}$.

\subsection{Effect of Magnesium-Based Layered Composite on Flame Retardance of EP}

Green synthesized LDHs in 2.1 were added to EP. The flame retardance of EP was evaluated from two aspects of different LDHs content and different anionic intercalated LDHs. LDHs are composed of the layered structure of magnesium hydroxide $\left(\mathrm{M}^{2+}(\mathrm{OH})_{2}\right)$ [10]. LDHs can provide good flame retardant and smoke suppression performance due to its unique chemical composition and layered structure [11]. Fig. 3 shows the structure of LDHs.

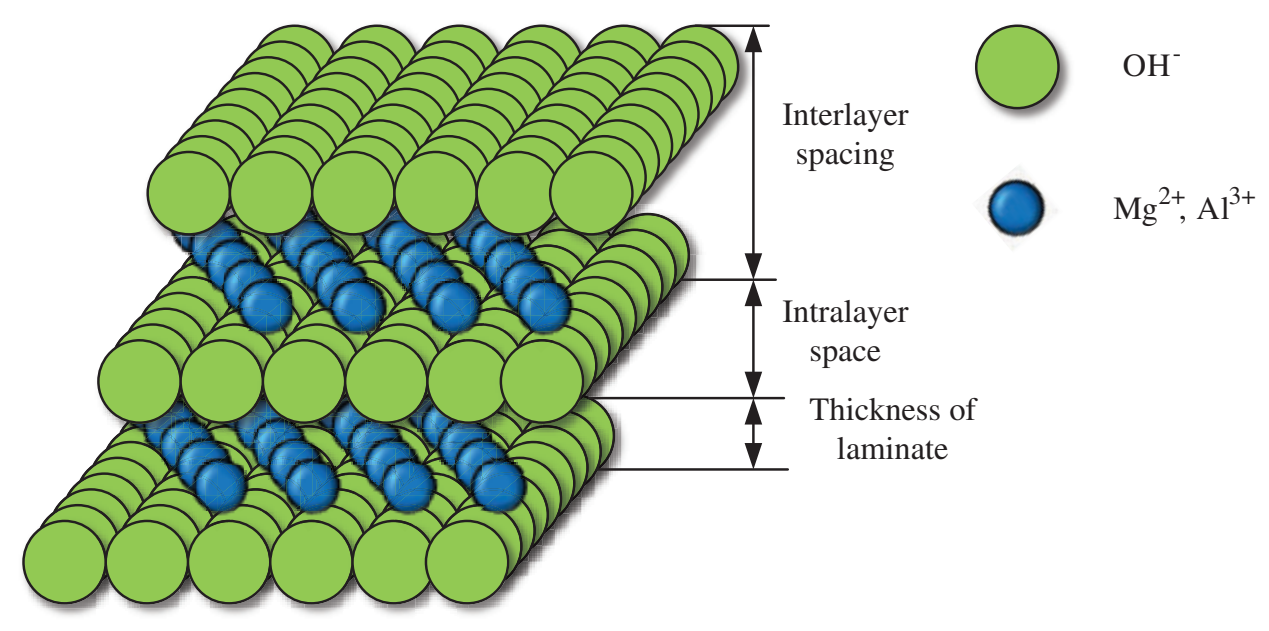

Figure 3: Structural diagram of LDHs

(1) Experimental materials and instruments

Table 1 presents the experimental raw materials used in this experiment.

Table 1: Specifications and manufacturers of experimental raw materials

\begin{tabular}{lll}
\hline Experimental raw materials & Specifications & Manufacturers \\
\hline $\mathrm{MgAl}-\mathrm{NO}_{3}$-LDHs & Analytical Reagent & Laboratory self-made \\
$\mathrm{MgAl}-\mathrm{Cl}-\mathrm{LDHs}$ & Analytical Reagent & Laboratory self-made \\
$\mathrm{MgAl}_{\mathrm{SO}}$-LDHs & Analytical Reagent & Laboratory self-made \\
$\mathrm{m}-\mathrm{PDA}$ & Analytical Reagent & $\begin{array}{l}\text { Shanghai Macklin Biochemical Co., Ltd. } \\
\text { (Shanghai, China) }\end{array}$ \\
$\mathrm{E}-44$ & Industrial grade & $\begin{array}{l}\text { Baling Petrochemical Co., Ltd. } \\
\text { (Yueyang, China) }\end{array}$ \\
\hline
\end{tabular}


Table 2 shows the instruments used in this experiment.

Table 2: Specifications and manufacturers of experimental instruments

\begin{tabular}{|c|c|c|}
\hline Experimental instruments & Specifications & Manufacturers \\
\hline Electronic analytical balance & BSA224S-CW & $\begin{array}{l}\text { Sartorius Instrument Co., Ltd. (Shanghai, } \\
\text { China) }\end{array}$ \\
\hline $\begin{array}{l}\text { Constant temperature magnetic } \\
\text { stirrer }\end{array}$ & DF-101S & $\begin{array}{l}\text { Gongyi Yuhua Instrument Co., Ltd. } \\
\text { (Gongyi, China) }\end{array}$ \\
\hline Electric blast drying oven & GZX-9070MBE & $\begin{array}{l}\text { Shanghai Boxun Industrial Co., Ltd. } \\
\text { (Shanghai, China) }\end{array}$ \\
\hline Polytetrafluoroethylene mould & Standard mould & $\begin{array}{l}\text { Shenzhen Huangrun hardware and } \\
\text { plastic products Co., Ltd. (Shenzhen, China) }\end{array}$ \\
\hline Synchronous thermal analyzer & $\begin{array}{l}\text { DTG/DSC3+ TG- } \\
\text { DSC }\end{array}$ & METTLER TOLEDO \\
\hline Oxygen index tester & JF-3 & $\begin{array}{l}\text { Nanjing Jiangning Analytical Instrument } \\
\text { Co., Ltd. (Nanjing, China) }\end{array}$ \\
\hline Cone calorimeter & FTT0007 & Fire Testing Technology \\
\hline
\end{tabular}

(2) Experimental methods

Fig. 4 displays the preparation process of EP-LDHs composites.

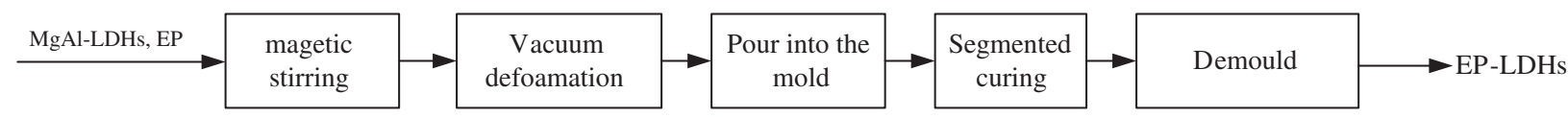

Figure 4: Preparation flow chart of EP-LDHs composites

Fig. 4 shows that the preparation process of EP-LDHs composite was as follows. EP was placed in the oven for $60 \mathrm{~min}$ at $50^{\circ} \mathrm{C}$; then, $40 \mathrm{~g}$ of preheated $\mathrm{EP}$ was taken into a beaker and placed in a $65^{\circ} \mathrm{C}$ water bath pan; $3.5 \mathrm{~g}$ m-phenylenediamine was weighted as curing agent, added to EP and stirred for $8 \mathrm{~min}$. Then, EP was vacuumed in a vacuum drying oven at $60^{\circ} \mathrm{C}$ for $20 \mathrm{~min}$; the polytetrafluoroethylene mold was preheated and coated with a small amount of silicone oil as the release agent; next, the defoamined EP was poured into the mold and pre-cured for $2 \mathrm{~h}$ at $80^{\circ} \mathrm{C}$. Then, the mold was cured at $120^{\circ} \mathrm{C}$ for $3 \mathrm{~h}$, and then cooled to room temperature naturally and demoulded.

The effects of different anion intercalations on EP flame retardant properties were investigated by adding $10 \mathrm{wt} \%, 15 \mathrm{wt} \%$ and $30 \mathrm{wt} \% \mathrm{MgAl}-\mathrm{NO}_{3}$-LDHs, as well as $30 \mathrm{wt} \% \mathrm{MgAl}-\mathrm{NO}_{3}$-LDHs and MgAl-Cl-LDHs and $\mathrm{MgAl}_{-\mathrm{SO}_{4}}$-LDHs to EP matrix, respectively. Fig. 5 reveals the composition of different EP-LDHs composites.

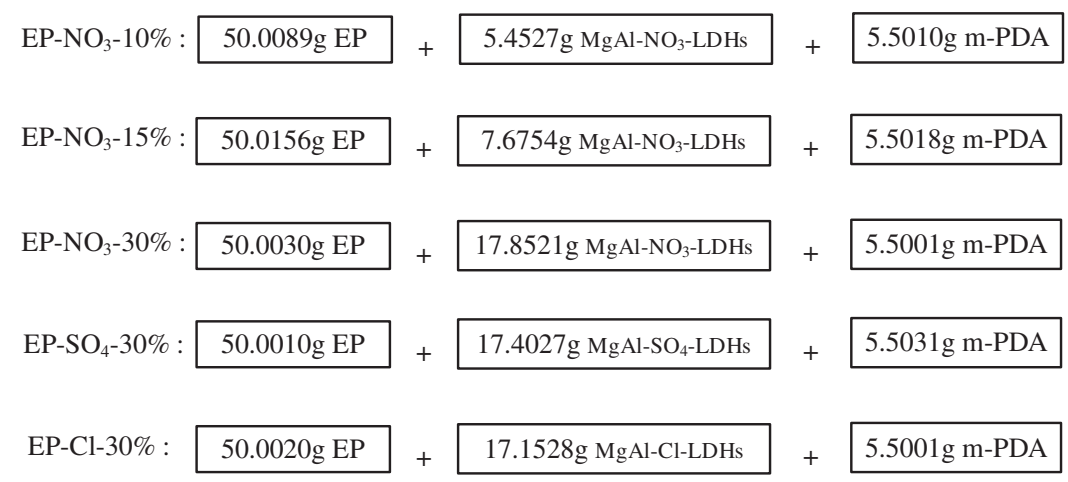

Figure 5: Composition of different EP-LDHs composite samples 


\section{(3) Experimental characterization means}

The composition and thermal stability of EP-LDHs composites were analyzed by the synchronous thermal analyser (TG-DTG). The determination conditions of thermogravimetric analysis were as follows. $5 \sim 10 \mathrm{mg}$ sample was taken, the atmosphere was air, the gas flow rate was $50 \mathrm{~mL} \cdot \mathrm{min}^{-1}$, the temperature range was $20^{\circ} \mathrm{C} \sim 80^{\circ} \mathrm{C}$, and the heating rate was $20^{\circ} \mathrm{C} \cdot \mathrm{min}^{-1}$.

The LOI of EP-LDHs composites was determined by the LOI method. The calculation equation of LOI is as follows:

In (1), $\left[\mathrm{O}_{2}\right]$ was the flow rate of oxygen, $\mathrm{L} \cdot \mathrm{min}^{-1}$, and $\left[\mathrm{N}_{2}\right]$ was the flow rate of nitrogen. Table 3 shows the specific flammable conditions.

Table 3: LOI index flammability situation

\begin{tabular}{ll}
\hline LOI index & Flammability \\
\hline$<21 \%$ & The material is flammable \\
$22 \% \sim 26 \%$ & The material is self-extinguishing \\
$>27 \%$ & The material is difficult to burn \\
\hline
\end{tabular}

The determination conditions were as follows: spline size $=130 \mathrm{~mm} \times 6.5 \mathrm{~mm} \times 3.2 \mathrm{~mm}$. It was ensured that the pressure of nitrogen and oxygen was less than $0.03 \mathrm{MPa}$, and the combustion system was flushed for $30 \mathrm{~s}$; a line $50 \mathrm{~mm}$ away from the ignition end on the wide surface of the sample was drawn. The lower end of the sample was clamped and placed in the combustion column. The ignition source was removed immediately after the sample was ignited with propane, and the minimum volume fraction of $\mathrm{O}_{2}$ in the mixture of $\mathrm{N}_{2}$ and $\mathrm{O}_{2}$ when the sample continues to burn to $50 \mathrm{~mm}$ mark was recorded.

Cone calorimeter was the most commonly used method to characterize the combustion performance of polymer materials. The essence of cone calorimetry was to analyze the oxygen consumption in the process of material combustion and obtain the heat released by combustion. It was known to consume $1 \mathrm{~g}$ of oxygen and release $13.1 \mathrm{~kJ}$ of heat. The curves for analyzing the flame retardant properties of materials during cone calorimetry test included heat release rate (HHR), total heat release, mass loss rate, effective combustion heat, specific extinction area, smoke generation rate, total smoke generation, and the generation of carbon monoxide and carbon dioxide. In this experiment, HHR curve was adopted to characterize and analyze the flame retardant properties of the materials. Cone calorimetry was employed to test cone calorimetric combustion of EP-LDHs composites. The test conditions were as follows. The ambient temperature was $23^{\circ} \mathrm{C}$, the ambient pressure was $101.68 \mathrm{kPa}$, the relative humidity was $50 \%$, the $\mathrm{C}$ coefficient was 0.04299 , the thermal radiation power was $50 \mathrm{~kW} \cdot \mathrm{m}^{-2}$, and the sample size was $100 \mathrm{~mm} \times 100 \mathrm{~mm} \times$ $3 \mathrm{~mm}$. Sample preparation: the sample to be tested was wrapped with aluminum foil; the bottom and side of the sample were covered, so that the upper surface of the sample was completely exposed in the air; then, the wrapped sample was placed in the sample rack.

LOI $=\frac{\left[\mathrm{O}_{2}\right]}{\left[\mathrm{O}_{2}\right]+\left[\mathrm{N}_{2}\right]} \times 100 \%$

\section{Green Synthesis of Magnesium-Based Layered Composites and Its Effect on Flame Retardance}

\subsection{Analysis of Green Synthesis Results of MgAl-LDHs}

$\mathrm{Al}\left(\mathrm{NO}_{3}\right)_{3} \cdot 9 \mathrm{H}_{2} \mathrm{O}$ was taken as the aluminum source, $\mathrm{n}_{\mathrm{Mg}}: \mathrm{n}_{\mathrm{Al}}=3: 1 \sim 4: 1$, and the reaction was carried out for $12 \mathrm{~h}$. Fig. 6 presents the XRD of the obtained MgAl-NO ${ }_{3}-\mathrm{LDHs}$. 


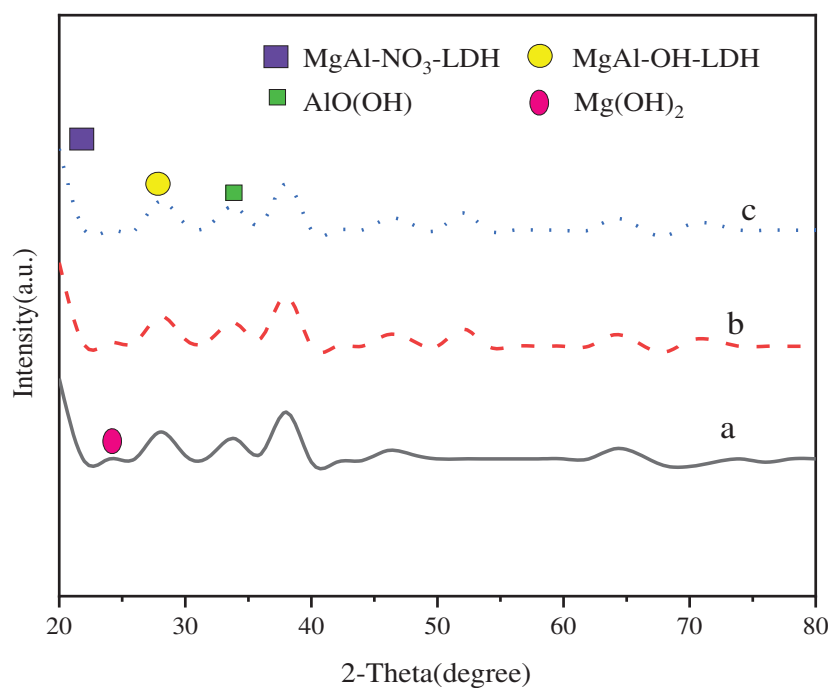

Figure 6: XRD patterns of $\mathrm{MgAl}-\mathrm{NO}_{3}$-LDHs during 3:1 4:1 (a) $\mathrm{Mg}: \mathrm{Al}=3: 1$, (b) $\mathrm{Mg}: \mathrm{Al}=3.5: 1$, (c) $\mathrm{Mg}$ : $\mathrm{Al}=4: 1$

Fig. 6 reveals that the characteristic diffraction peaks with sharp front, high intensity and decreasing intensity appear in all samples in $2 \theta=20^{\circ}$ and $30^{\circ}$, indicating that $\mathrm{NO}_{3}{ }^{-}$intercalated $\mathrm{MgAl}-\mathrm{LDHs}$ are obtained. There are impurities in three groups of samples when $\mathrm{n}_{\mathrm{Mg}}: \mathrm{n}_{\mathrm{Al}}=3: 1 \sim 4: 1$. The characteristic diffraction peak of $\mathrm{AlO}(\mathrm{OH})$ is obvious at the peak when $\mathrm{Mg}: \mathrm{Al}=3: 1$, at $2 \theta=15^{\circ}$; the characteristic diffraction peak of $\mathrm{AlO}(\mathrm{OH})$ disappears when $\mathrm{Mg}: \mathrm{Al}=4: 1$, but multiple peaks appear in $2 \theta=11^{\circ}$ and $21^{\circ}$, indicating that magnesium hydroxide is excessive.

Therefore, the characteristic diffraction peaks of $\mathrm{AlO}(\mathrm{OH})$ gradually weaken to disappear with the increase of $\mathrm{Mg}$ : $\mathrm{Al}$ molar ratio, and the characteristic diffraction peaks of $\mathrm{Mg}(\mathrm{OH})_{2}$ begin to appear.

Fig. 7 displays the XRD patterns when the molar ratio of $\mathrm{Mg}: \mathrm{Al}$ is further set to 3.2:1 and 3.4:1.

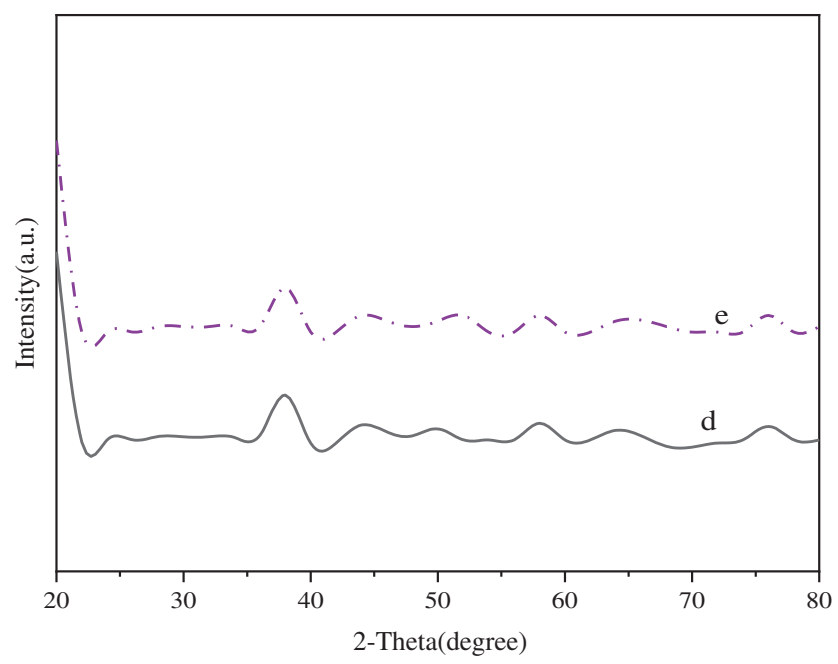

Figure 7: XRD patterns of $\mathrm{MgAl}-\mathrm{NO}_{3}$-LDHs during 3.2:1 3.4:1 d. $\mathrm{Mg}: \mathrm{Al}=3.2: 1$, e. $\mathrm{Mg}: \mathrm{Al}=3.4: 1$ 
Fig. 7 suggests that the characteristic diffraction peaks of $\mathrm{MgAl}-\mathrm{OH}-\mathrm{LDHs}$ and $\mathrm{Mg}(\mathrm{OH})_{2}$ can be detected when $\mathrm{n}_{\mathrm{Mg}}: \mathrm{n}_{\mathrm{Al}}=3.4: 1$; when $\mathrm{n}_{\mathrm{Mg}}: \mathrm{n}_{\mathrm{Al}}=3.2: 1$, the baseline of MgAl-OH-LDHs is stable and the front is sharp and symmetrical.

The results show that $\mathrm{MgAl}-\mathrm{NO}_{3}$-LDHs with the single crystal phase is obtained when the $\mathrm{Mg}: \mathrm{Al}$ molar ratio is $3.2: 1$.

\subsection{Effect of MgAl-LDHs on Flame Retardance of EP}

(1) Thermal analysis of $\mathrm{MgAl}-\mathrm{NO}_{3}$-LDHs with different addition

$10 \mathrm{wt} \%, 15 \mathrm{wt} \%$ and $30 \mathrm{wt} \% \mathrm{MgAl}-\mathrm{NO}_{3}$-LDHs are added to the EP matrix respectively to explore the effect of different amounts of LDHs on the flame retardance of EP. Fig. 8 shows the thermogravimetric curves (TG) of EP-LDHs composites with different $\mathrm{MgAl}-\mathrm{NO}_{3}$-LDHs contents.
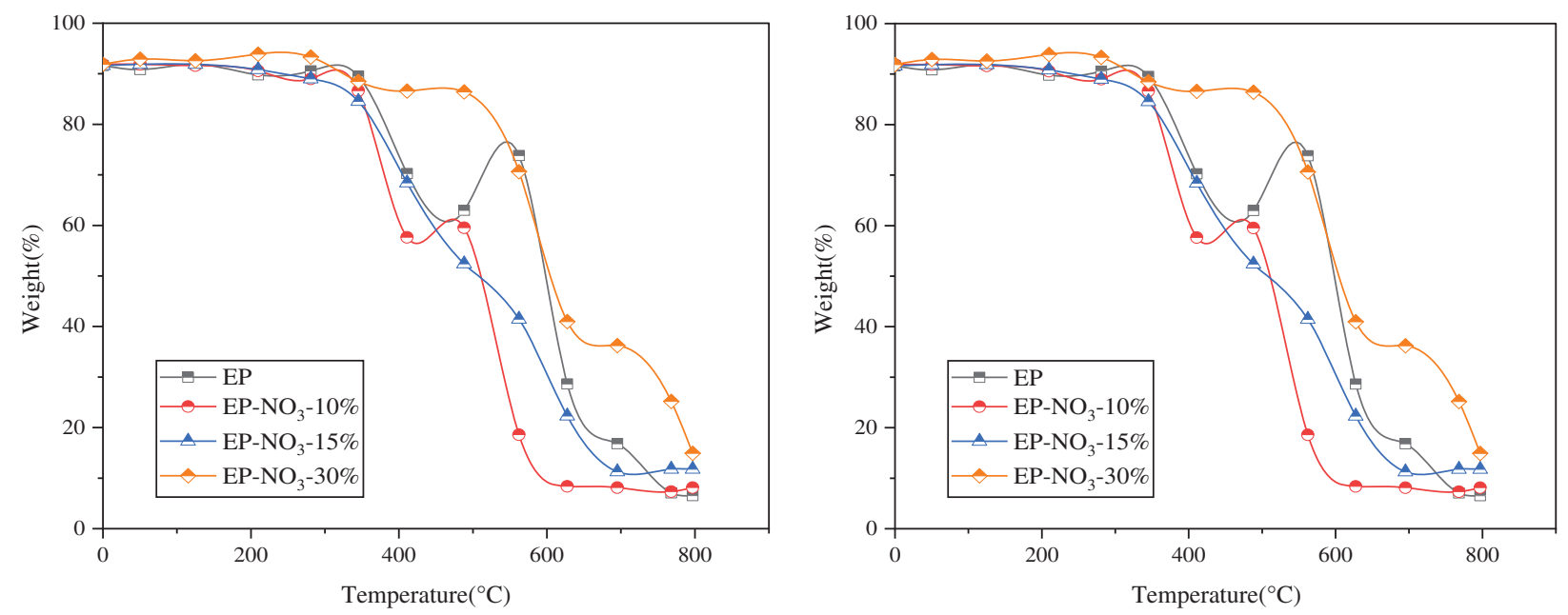

Figure 8: TG curve of EP-LDHs with different $\mathrm{MgAl}-\mathrm{NO}_{3}-\mathrm{LDHs}$ addition

Fig. 8 reveals that the decomposition of pure EP and EP-LDHs composites can be divided into two stages at $30^{\circ} \mathrm{C} \sim 800^{\circ} \mathrm{C}$. The first stage is the formation of an unstable carbon layer due to the cleavage of the EP molecular chain. The maximum thermal decomposition rate Tmax is about $390^{\circ} \mathrm{C}$; in the second stage, the unstable carbon layer decomposes further due to the increase of temperature. The maximum thermal decomposition rate appears at $\operatorname{Tmax} 2=571^{\circ} \mathrm{C}$.

The decrease of thermal stability of EP may be due to the incomplete dispersion of LDHs in the EP matrix. On the other hand, with the addition of LDHs, the residual carbon content of EP- $\mathrm{NO}_{3}-10 \%$, EP$\mathrm{NO}_{3}-15 \%$ and $\mathrm{EP}-\mathrm{NO}_{3}-30 \%$ increases after the end of the reaction, indicating that $\mathrm{MgAl}-\mathrm{NO}_{3}-\mathrm{LDHs}$ can promote the carbon formation of EP during the heating process, thus improving the flame retardance.

(2) Analysis of LOI of MgAl- $\mathrm{NO}_{3}$-LDHs based on different additives

Fig. 9 is the LOI diagram of EP-LDHs composites with different $\mathrm{MgAl}-\mathrm{NO}_{3}$-LDHs contents.

Fig. 9 shows that the LOI value of pure EP solid content is $25.4 \%$. The LOI of EP-LDHs increases to $28.3 \%, 29.1 \%$ and $29.6 \%$ when the LDHs content is $10 \%, 15 \%$ and $30 \%$, respectively.

This indicates that higher $\mathrm{O}_{2}$ concentration is needed to ignite EP-LDHs composites and maintain stable combustion. Hence, the addition of $\mathrm{MgAl}-\mathrm{NO}_{3}-\mathrm{LDHs}$ to EP matrix has certain flame retardance.

(3) Cone calorimetric analysis of $\mathrm{MgAl}-\mathrm{NO}_{3}-\mathrm{LDHs}$ with different additions 
FDMP, 2022, vol.18, no.3

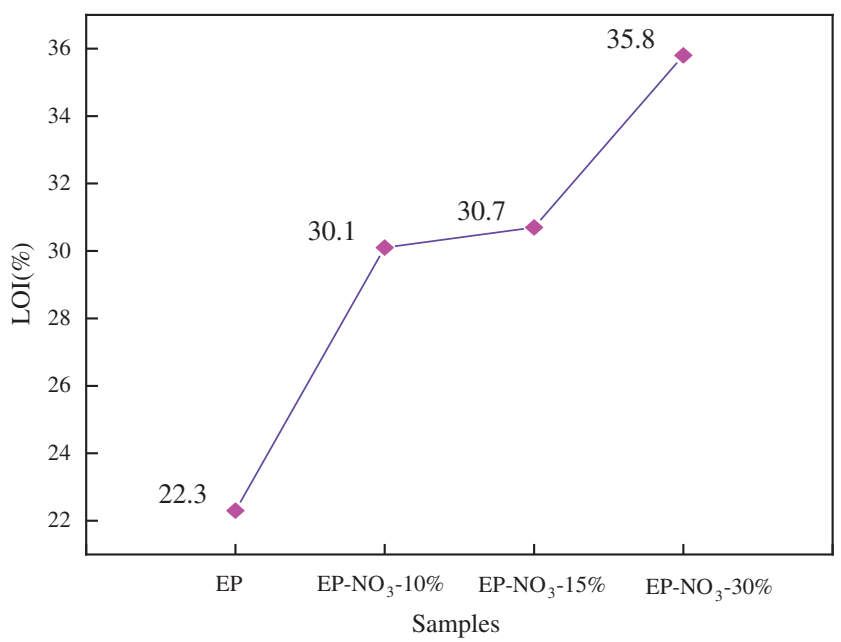

Figure 9: LOI of EP-LDHs prepared with different $\mathrm{MgAl}-\mathrm{NO}_{3}-\mathrm{LDHs}$ additions

Fig. 10 displays the time-heat release rate (HRR) curves of EP-LDHs prepared with different MgAl-NO ${ }_{3}-\mathrm{LDHs}$ additions.

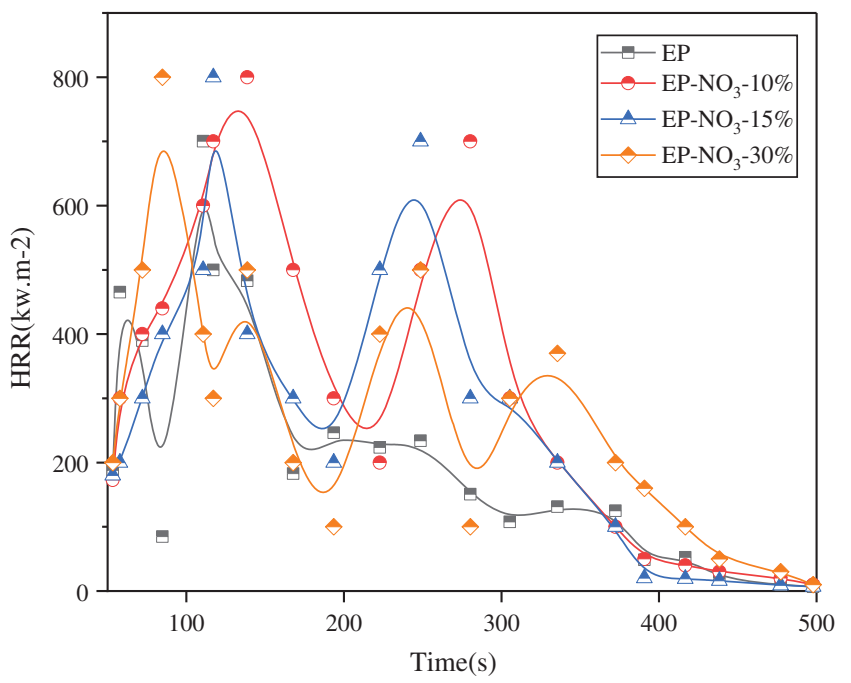

Figure 10: HRR curves of EP-LDHs prepared with different $\mathrm{MgAl}-\mathrm{NO}_{3}-\mathrm{LDHs}$ additions

Fig. 10 suggests that the maximum heat release rate of pure EP (PHRR) is $1250.07 \mathrm{~kW} \cdot \mathrm{m}^{-2}$. The PHRR

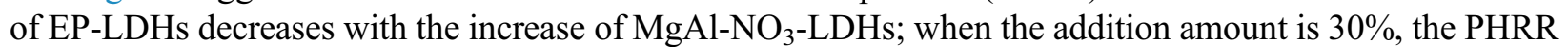
value is the lowest, $932.60 \mathrm{~kW} \cdot \mathrm{m}^{-2}$, which is $25.40 \%$ lower than that of pure EP.

Therefore, the addition of $\mathrm{MgAl}-\mathrm{NO}_{3}-\mathrm{LDHs}$ to the EP matrix has a certain flame retardance, and the more the amount is added, the better the flame retardancy is.

(4) Thermal analysis of MgAl-LDHs based on different anions intercalations

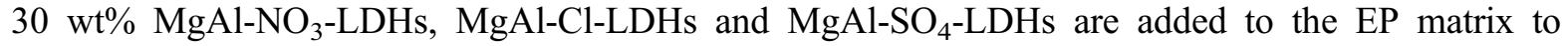
investigate the effect of MgAl-LDHs with different anionic intercalation on the flame retardance of EP. Fig. 11 shows the thermogravimetric (TG) curves of EP-LDHs composites prepared by MgAl-LDHs intercalated with different anions. 


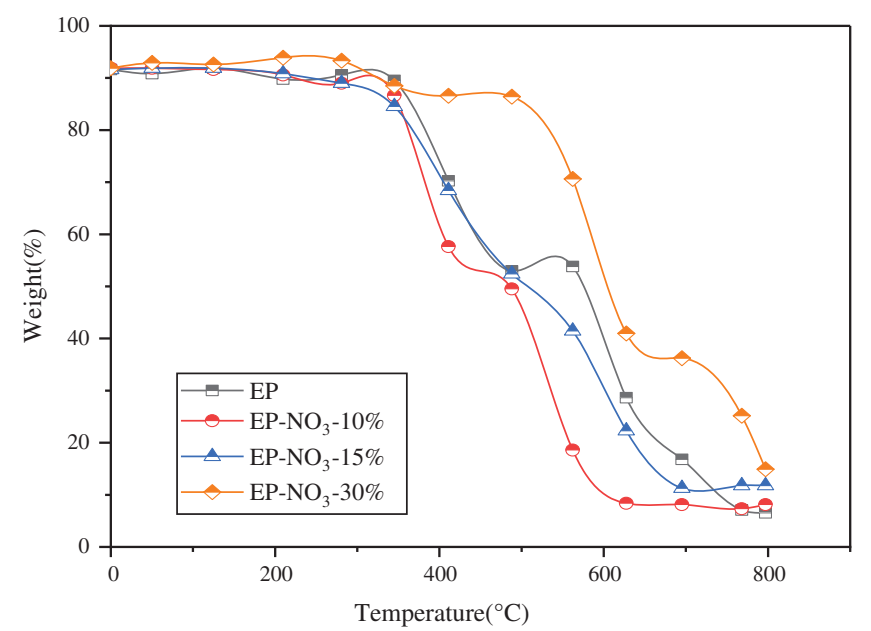

Figure 11: TG curve of EP-LDHs composite prepared by MgAl-LDHs with different anion intercalations

Fig. 11 suggests that different EP-LDHs composites are divided into two decomposition processes in the heating process. The initial cracking temperature $\mathrm{T} 5 \%$ of pure $\mathrm{EP}$ is $381.3^{\circ} \mathrm{C}$, while the $\mathrm{T} 5 \%$ of $\mathrm{MgAl}-\mathrm{NO}_{3^{-}}$ $30 \%, \mathrm{MgAl}-\mathrm{Cl}-30 \%$ and $\mathrm{MgAl}-\mathrm{SO}_{4}-30 \%$ decreases to $357.1^{\circ} \mathrm{C}, 371.3^{\circ} \mathrm{C}$ and $367.7^{\circ} \mathrm{C}$, respectively, with a decrease of less than $6 \%$.

Therefore, it can be concluded that the addition of three EP-LDHs will slightly reduce the stability of EP.

(5) Analysis of LOI of MgAl-LDHs intercalated with different anions

Fig. 12 shows the LOI values of EP-LDHs composites prepared by MgAl-LDHs intercalated with different anions.

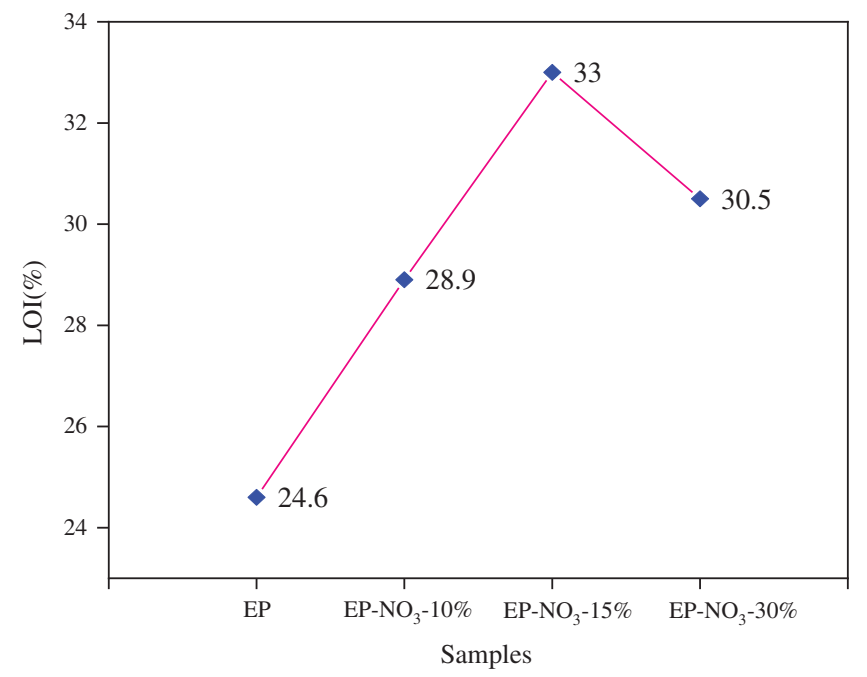

Figure 12: LOI values of EP-LDHs composites prepared by MgAl-LDHs intercalated with different anions

Fig. 12 reveals that the LOI values of pure EP solid content are $25.4 \%$, while that of $\mathrm{MgAl}-\mathrm{NO}_{3}-30 \%$, $\mathrm{MgAl}-\mathrm{Cl}-30 \%$ and $\mathrm{MgAl}_{-} \mathrm{SO}_{4}-30 \%$ are $29.5 \%, 33.2 \%$ and $28.1 \%$, respectively. Unlike pure EP, the LOI value of the three EP-LDHs composites is more than $27 \%$, which meets the standard of fire resistance. 
The increase of LOI value indicates that all three kinds of MgAl-LDHs have the certain flame retardant ability in the EP matrix.

(6) Cone calorimetric analysis of MgAl LDHs based on different anion intercalations

Fig. 13 displays the time-HRR curve of EP-LDHs composites prepared by MgAl-LDHs with different anion intercalations.

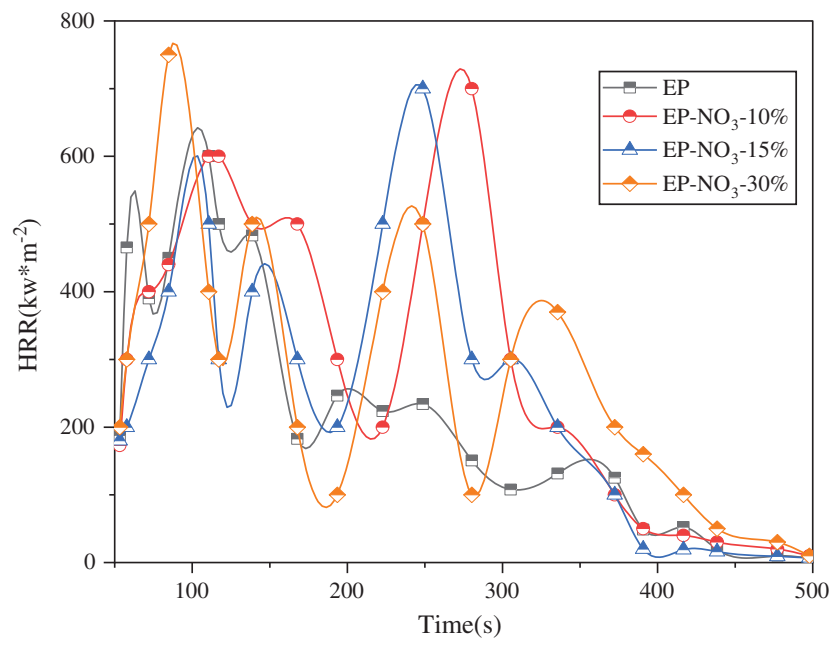

Figure 13: HRR curve of EP-LDHs prepared by MgAl-LDHs intercalated with different anions

Fig. 13 reveals that the PHRR value of pure EP is $1250.07 \mathrm{~kW} \cdot \mathrm{m}^{-2}$, and the PHRR value of EP-LDHs composites decreases in varying degrees after three kinds of anionic intercalated $\mathrm{MgAl}$-LDHs are added into EP. Among them, the PHRR values of MgAl- $-\mathrm{NO}_{3}-30 \%, \mathrm{MgAl}-\mathrm{Cl}-30 \%$ and $\mathrm{MgAl}-\mathrm{SO}_{4}-30 \%$ decreases to $932.60,947.64$ and $965.32 \mathrm{~kW} \cdot \mathrm{m}^{-2}$, respectively, with a decrease rate of $25.40 \%, 24.19 \%$ and $22.79 \%$, respectively. Among them, the PHRR of $\mathrm{MgAl}-\mathrm{NO}_{3}-30 \%$ decreases the most.

Therefore, $\mathrm{MgAl}-\mathrm{NO}_{3}$ - $\mathrm{LDHs}$ has the best flame retardance in the EP matrix among the three kinds of MgAl-LDHs.

Finally, the results are summarized. In the experiment of green synthesis of magnesium matrix-based layered composites, $\mathrm{MgAl}-\mathrm{NO}_{3}-\mathrm{LDHs}$ with single crystal phase is obtained when the $\mathrm{Mg}: \mathrm{Al}$ molar ratio is 3.2:1; the experimental results of adding 10, 15 and $30 \mathrm{wt} \% \mathrm{MgAl}-\mathrm{NO}_{3}-\mathrm{LDHs}$ to EP matrix show that $\mathrm{MgAl}-\mathrm{NO}_{3}$-LDHs can promote the carbon formation of EP during heating, so as to improve the flame retardant performance; the results of LOI analysis based on different addition amounts of $\mathrm{MgAl}^{-\mathrm{NO}_{3}-}$ LDHs show that $\mathrm{MgAl}-\mathrm{NO}_{3}-\mathrm{LDHs}$ added to EP matrix have certain flame retardant properties; the more the $\mathrm{MgAl}-\mathrm{NO}_{3}-\mathrm{LDHs}$ is added, the better the flame retardancy is. Moreover, adding $\mathrm{MgAl}-\mathrm{NO}_{3}-\mathrm{LDHs}$ to EP matrix has certain smoke suppression performance. The more the amount is, the better the smoke suppression performance is; the experimental results of thermal energy analysis based on MgAl-LDHs intercalated with different anions show that during the heating process, the LDHs intercalated with three different anions can promote the carbon formation efficiency of EP, so as to improve the flame retardancy of EP; the results of LOI analysis of MgAl-LDHs intercalated with different anions show that MgAl$\mathrm{NO}_{3}-30 \%, \mathrm{MgAl}-\mathrm{Cl}-30 \%$ and $\mathrm{MgAl}-\mathrm{SO}_{4}-30 \%$ have certain flame retardancy in EP matrix; the cone calorimetric analysis results of $\mathrm{MgAl}-\mathrm{LDHs}$ based on different anion intercalations show that among the three $\mathrm{MgAl}-\mathrm{LDHs}, \mathrm{MgAl}-\mathrm{NO}_{3}-\mathrm{LDHs}$ has the best flame retardant performance in EP matrix. 


\section{Conclusion}

Magnesium-based LDHs are green synthesized by a new method and added to the EP matrix. The effect of magnesium-based LDHs on the flame retardance of EP is evaluated. The main conclusions are as follows. First, the optimum technological conditions for the green synthesis of magnesium-based LDHs are as follows. The molar ratio of $\mathrm{Mg}: \mathrm{Al}$ is 3.2:1; then, the green synthesis of the magnesium-based layered composite can reduce the thermal stability of EP and inhibit the heat and smoke release of EP; finally, the flame retardance of $\mathrm{MgAl}-\mathrm{NO}_{3}-\mathrm{LDHs}$ intercalated with nitrate ion in EP matrix is the best. This exploration still has the following two limitations. First, MgAl-LDHs are directly added to EP, which may lead to the uneven dispersion of MgAl-LDHs in the EP matrix. In the follow-up study, MgAl-LDHs can be modified and then added to EP to obtain better flame retardance. Second, the mechanical properties of EP are affected by adding flame retardant to EP. In the follow-up study, the mechanical properties of EP-LDHs can be further studied. The flame retardant properties of EP are evaluated based on the green synthesis of magnesium-based layered composites. The research content provides a certain reference for the flame retardant method of EP, and also provides a new green way for the synthesis of magnesium-based layered composites.

Funding Statement: The authors received no specific funding for this study.

Conflicts of Interest: The authors declare that they have no conflicts of interest to report regarding the present study.

\section{References}

1. Akinwamide, S. O., Lemika, S. M., Obadele, B. A., Akinribide, O. J., Abe, B. T. et al. (2019). A study on microstructural and mechanical properties of a stir cast $\mathrm{Al}$ (SiC-Mg-TiFe) composite. Fluid Dynamics \& Materials Processing, 15(1), 15-26. DOI 10.32604/fdmp.2019.04761.

2. Baran, A., Polański, M. (2020). Magnesium-based materials for hydrogen storage-A scope review. Materials (Basel), 13(18), 3993. DOI 10.3390/ma13183993.

3. Leonés, A., Lieblich, M., Benavente, R., Gonzalez, J. L., Peponi, L. (2020). Potential applications of magnesiumbased polymeric nanocomposites obtained by electrospinning technique. Nanomaterials, 10(8), 1524. DOI 10.3390/nano10081524.

4. Yeboah, M. L., Li, X., Zhou, S. (2020). Facile fabrication of biochar from palm kernel shell waste and its novel application to magnesium-based materials for hydrogen storage. Materials, 13(3), 625. DOI 10.3390/ma13030625.

5. Venkatraman, S. K., Swamiappan, S. (2020). Review on calcium and magnesium-based silicates for bone tissue engineering applications. Journal of Biomedical Materials Research Part A, 108(7), 1546-1562. DOI 10.1002/ jbm.a.36925.

6. Liu, C., Du, Y., Liang, J., Meng, H., Zhang, P. (2020). Large eddy simulation of gasoline-air mixture explosion in long duct with branch structure. Fluid Dynamics \& Materials Processing, 16(3), 537-547. DOI 10.32604/fdmp.2020.09119.

7. Li, G. L. (2020). Simulation of the thermal environment and velocity distribution in a lecture hall. Fluid Dynamics \& Materials Processing, 16(3), 549-559. DOI 10.32604/fdmp.2020.09219.

8. Netkueakul, W., Fischer, B., Walder, C., Nüesch, F., Wang, J. (2020). Effects of combining graphene nanoplatelet and phosphorous flame retardant as additives on mechanical properties and flame retardancy of epoxy nanocomposite. Polymers, 12(10), 2349. DOI 10.3390/polym12102349.

9. Wang, J., Liu, Y., Guo, X., Qu, H., Ma, J. (2020). Efficient adsorption of dyes using polyethyleneimine-modified nh 2-mil-101(Al) and its sustainable application as a flame retardant for an epoxy resin. ACS Omega, 5(50), 32286-32294. DOI 10.1021/acsomega.0c04118.

10. Li, L., Cai, Z. (2020). Flame-retardant performance of transparent and tensile-strength-enhanced epoxy resins. Polymers, 12(2), 317. DOI 10.3390/polym12020317.

11. Song, K., Wang, Y., Fang, R., Yang, W., Liu, J. (2020). Synthesis of a novel spirocyclic inflatable flame retardant and its application in epoxy composites. Polymers, 12(11), 2534. DOI 10.3390/polym12112534. 